\title{
Asociación entre el propósito de vida y los factores socioeconómicos de excombatientes de las Farc-EP en Chalán y Colosó, Colombia
}

\begin{abstract}
Association between the purpose of life and the socioeconomic factors of ex-combatants of the Farc-EP in Chalán and Colosó, Colombia
\end{abstract}

\author{
Luisa F. Martinez-Ballutt ${ }^{*}$ (B) ; Olga-Leonor Hernández-Bustamante ${ }^{(\mathbb{D}}$; Maria L. Vergara Alvarez ${ }^{3}$ (D)
}

\section{RESUMEN}

Objetivo. La presente investigación se centró en conocer la relación que tiene el propósito de vida y los factores socioeconómicos de los excombatientes de las Farc-EP residentes en los municipios de Colosó y Chalán, Colombia. Método. De tipo correlacional, diseño no experimental de corte transversal. Se utilizó la Escala Dimensional del Sentido de Vida (EDSV) y una serie de preguntas ad hoc construidas por los investigadores para la indagación de por factores socioeconómicos Resultados. Los resultados obtenidos nos muestran que un $40 \%$ de los sujetos participantes, excombatientes de las Farc-EP, se sitúan en el nivel medio sentido vital. Conclusiones. Los aspectos socioeconómicos que se encontraron asociados al propósito vital fueron de tipo educativo y tiempo de reintegración de cada excombatiente a la vida civil.

Palabras claves: Escala Dimensional Sentido de Vida; Excombatiente; Farc-EP; Propósito de Vida.

\begin{abstract}
Objective. This research focused on knowing the relationship between the purpose of life and the socioeconomic factors of the ex-combatants of the Farc-EP residing in the municipalities of Colosó and Chalán, Colombia. Method. Correlational type, non-experimental cross-sectional design. The Dimensional Scale of the Meaning of Life (EDSV or DSML) and a series of ad hoc questions constructed by the researchers were used to investigate by socioeconomic factors. Results. The results obtained show us that $40 \%$ of the participating subjects, ex-combatants of the Farc-EP, are situated in the medium level vital sense. Conclusions. The socioeconomic aspects that were found associated with the vital purpose were educational and time of reintegration of each excombatant to civil life.
\end{abstract}

Keywords: Dimensional Scale Sense of Life; Ex-combatant; Farc-EP; Purpose of Life.

\footnotetext{
${ }^{1}$ Corporación Universitaria del Caribe - CECAR, Sincelejo, Colombia. luisa.martinezb@cecar.edu.co

${ }^{2}$ Corporación Universitaria del Caribe - CECAR, Grupo de investigación Dimensiones Humanas - Líneas Desarrollo cognitivo, salud mental y neuropsicología y Vulnerabilidad social y grupos poblacionales. Sincelejo, Colombia. olga.hernandezb@cecar.edu.co

${ }^{3}$ Corporación Universitaria del Caribe - CECAR, Sincelejo, Colombia. maria.vergaraa@cecar.edu.co

Como citar (APA)

Martinez-Ballutt, L. F., Hernández-Bustamante, O. L., Vergara Alvarez, M. L. (2020). Asociación entre el Propósito de Vida y los Factores Socioeconómicos de Excombatientes de las Farc-EP en Chalán y Colosó, Colombia. Búsqueda, v. 7, n. 25, e513. https://doi.org/10.21892/01239813.513
} 


\section{INTRODUCCIÓN}

Colombia ha sido, por más de medio siglo, un país en conflicto, de múltiples ataques contra la población civil por parte grupos armados al margen de la ley. Los intentos por parte del Estado de acabar con el conflicto armado se han convertido en más sangre que en un acercamiento a la Paz; esto y el narcotráfico, son causas indiscutibles del origen de la violencia que se ha vivido en Colombia (De la Espriella \& Vladimir, 2009).

Como ya es sabido, el país ha tratado de superar estos días de violencia y silenciar las armas con acuerdos. El último de ellos recién fue firmado en el 2016, suscrito entre las Farc y el Gobierno. La firma del acuerdo no significa el fin de los conflictos sino un largo camino por recorrer. Entre estos, es fundamental el proceso de reintegración social, económica y política de excombatientes, pues es el proceso por el cual estas personas una vez han dejado las armas, se integran tanto en el sistema democrático como en la vida civil (Radiónica, 2017). Hace más de 30 años, la Organización de las Naciones Unidas creó unas bases para reintegrar al ejercicio de la ciudadanía a los excombatientes de cualquier grupo armado; a esto se le llamó Desarme, Desmovilización y Reintegración (DDR). Este proceso tiene el objetivo de reforzar y fomentar los procesos de paz, aumentar la confianza entre las partes involucradas y construir las actividades iniciales de recuperación y la estabilización.

En Colombia, en el gobierno de Álvaro Uribe Vélez (2002-2010) se elevó el número de desmovilizaciones de grupos armados, más que todo grupos paramilitares, quienes participaron en el Programa de Reintegración. Este programa, busca incorporar a la vida civil a las personas desmovilizadas de grupos irregulares y su objetivo principal es desarrollar competencias y habilidades en las personas desmovilizadas y sus entornos; asimismo, busca promover espacios para las acciones de reconciliación y convivencia, y aumentar la corresponsabilidad de los actores externos (De la Espriella \& Vladimir, 2009).

Dentro de la Agencia para la Reincorporación y la Normatividad (ARN), se encuentra la Ruta de Reintegración, entendida como el conjunto de estrategias, metodologías, beneficios y condiciones que se imparten en la persona en proceso de reintegración, que busca promover en ellas capacidades para superar la situación de vulnerabilidad y el ejercicio autónomo de la ciudadanía (Agencia Colombiana para la Reintegración, 2016b). El objetivo del diseño de esta Ruta es buscar que la persona tenga un esquema de actividades acorde con los beneficios económicos, sociales y jurídicos del Proceso de Reintegración y que aporten al propósito de vida de cada individuo, el cual es esencial para la ruta puesto que se busca que la persona materialice sus aspiraciones en la vida civil (Agencia Colombiana para la Reintegración, 2016).

Sin embargo, ¿cómo perciben los reinsertados estas políticas y programas que les brinda el Estado para su regreso a la vida civil y reconstrucción de su propósito de vida? Rendón (2017) afirma que el $76 \%$ de las personas que han entrado al proceso de reintegración no han vuelto a delinquir, no han retomado las armas y después de 6 años han podido autosostenerse; este logro permite afirmar que el país está listo para reincorporar a los excombatientes a una nueva vida. Asimismo, se ha evidenciado en las investigaciones las preocupaciones que tienen algunos excombatientes hacia las oportunidades que les brindan los programas y políticas en aspectos laborales, educativos, de vivienda, de reconciliación, como también hacia la atención psicológica brindada por la ACR (ACR, 2008; Mejía, 2014; Lara \& Delgado, 2010, 2016; Rodríguez, et al. 2010).

Estas preocupaciones manifestadas por los mismos excombatientes sobre la ayuda que les brindan los programas de reintegración son preocupantes para la tan trabajada búsqueda del bienestar y convivencia de la población: la Paz. La asistencia psicológica a estas personas suele ser clave para que estas no vuelvan a reincidir en los grupos armados ilegales; esta asistencia es un espacio para que la persona pueda adquirir capacidades que le permitan reconstruir su propósito de vida, la superación de la situación de vulnerabilidad y el proceso para el ejercicio autónomo de su vida civil. También, deben incluirse los valores asociados al trato humano en las dimensiones laboral, familiar y personal; además, suele ser difícil y en ocasiones resulta negativo para el excombatiente adentrarse a la vida civil y relacionarse con los ciudadanos; asimismo, 
suelen verse casos de estigma social y rechazo (Instituto de Estudios Geoestratégicos y Asuntos Políticos, 2013).

Según la Agencia Colombiana de Reintegración, 5.3\% de las 48 mil personas que han entrado en proceso de reintegración, ha sido asesinadas y el $9.3 \%$ recibe amenazas o enfrenta riesgos a su seguridad (Bravo, 2016). Asimismo, en el ámbito laboral, solo el 20\% de los desmovilizados encuentran un empleo formal y esto por cinco razones: (1) desconfianza por parte de las empresas, (2) los desmovilizados no están preparados, (3) antecedentes penales, (4) el autoempleo casi siempre es informal, (5) falta de motivación salarial (Rendón, 2018).

Sennett (2018) alguna vez expresó: "Nadie es capaz de construir una vida nueva si odia su pasado" (p. 117). De aquí se parte hacia la importancia de hablar sobre el propósito de vida de un reinsertado. Se comprende este propósito de vida como aquel conjunto de metas y valores de los individuos que se materializan en proyectos, alternativas y estilos de acción individual y colectiva (Rodríguez, Yunis \& Girón, 2015). Tal como lo expresan Rodríguez, Yunis \& Girón (2015) los excombatientes, al abandonar su entorno de guerra, su propósito de vida se ve altamente modificado, se mantienen los mismos valores y enseñanzas que tuvieron en la infancia, pero al transcurrir de entornos, su propósito de vida se altera por los intereses y percepciones que tienen sobre el nuevo contexto.

Según una investigación realizada por Rueda (2008) cada persona es una singularidad, por esto cada proceso de reintegración y su manera de retomar la vida civil es diferente. La subjetividad de cada excombatiente está ligada a la reconstrucción que haga a partir de sus sueños, propósitos y metas que le permitan construir una nueva identidad, olvidándose de la que le otorgaba el grupo armado e incluyendo las oportunidades que le puede brindar el nuevo contexto en el cual se desenvuelve (Rueda, 2008). En esta misma investigación se resaltan los comentarios que tienen varios excombatientes con respecto a su nueva visión de vida, afirmando que para ellos la educación y la familia son factores claves para las proyecciones que tienen a futuro sobre sus vidas, puesto que teniendo acceso a la educación crean capacidades que los ayudan a mejorar sus condiciones de vida y a su vez las de su familia.

Las variables anteriormente expuestas, sumando las desigualdades sociales, la pobreza, la cultura del inmediatismo económico al que se enfrentan en la nueva vida civil, entre otros aspectos socioeconómicos, afectan significativamente a los procesos de DDR y hace que sean más complejo alcanzar los objetivos propuestos por este mismo. Y, con esto, se obliga al Estado a diseñar nuevas estrategias, políticas y programas políticos que beneficien a ambos lados: por un lado, a la sociedad colombiana, mejorando sus condiciones generales, y, por el otro, brindando garantías específicas a quienes se desmovilizan, ayudando de ese modo a la reconstrucción de su propósito de vida en el marco de la legalidad (Mejía, 2014).

Frente a esta realidad, la logoterapia asiste a la búsqueda de sentido en la vida, así como también, un sentido a la misma adversidad (Noblejas de la Flor \& Rodríguez, 2005). En palabras de Frankl (1988) vivir quiere decir adquirir el compromiso de hallar la respuesta adecuada a todas las dificultades que esto representa y efectuar las obligaciones que la vida nos delega seguidamente. Este enfoque terapéutico fue desarrollado por Viktor Frankl para abordar el vacío existencial en las personas, lo cual para él es el problema psicológico más difícil de enfrentar en el mundo moderno y es producto de una carencia de significado en la vida, carencia que se caracteriza por el aburrimiento, desesperanza, depresión y pérdida de voluntad de vivir (Somaye, Sohrabi, Shahrak, Talepasand, Nokani \& Hasani, 2015).

Debido a la situación actual de nuestro país, sus intentos de alcanzar la paz, la creación de estas políticas y programas de reinserción, el concepto de propósito de vida y evidenciando la relación que tienen los factores familiares, educativos, laborales y sociales en los nuevos propósitos de vida de estos excombatientes, se hace necesario resaltar esta asociación, puesto que sería un punto de partida para posibles intervenciones o modificaciones en los programas impartidos para estas personas. Como también, a manera social, lograr ver al desmovilizado no como victimario sino como ser humano en búsqueda de un propósito luego de haber abandonado las armas como manera de lograr la supervivencia en el marco del abandono del Estado y de las escasas 
políticas sociales para la garantía de derechos de la población. Además, esta mirada humana a los excombatientes significa en sí misma un aporte para que esta población no se siga sintiendo tan estigmatizada en la sociedad y pueda fortalecer su propósito de vida.

Expuesto lo anterior, se hace necesario estudiar y conocer el sentido de vida de personas que se han retirado de las armas y que hoy en día ven la posibilidad de integrarse a vivir en sociedad, bajo condiciones totalmente diferentes; estudiar este aspecto es de relevancia para la sustentabilidad social y política de los acuerdos pactados por el país para alcanzar la paz, y desde una perspectiva psicosocial, el cambio de una vida en la guerra a una vida civil, puede incurrir cese de disparos y muertes, pero no incluye el sacrificio psicológico que hace la persona al abandonar una vida conflictiva, no logra encontrar un sentido a su existir en la nueva vida a la que se enfrenta (Matallana \& Villaneda, 2013). Por lo tanto, es importante destacar la importancia que tiene una reinserción integral de un desmovilizado a la sociedad, tomando en cuenta que luego de experimentar situaciones adversas y un choque por el enfrentamiento a la nueva vida en que se adaptan, se desestructura el sí mismo y cuando se modifica este sí mismo, se modifica el propósito de vida, por lo cual se hace importante revisar a nivel teórico los factores asociados al propósito vital de los excombatientes de las Farc-EP.

\section{METODOLOGÍA}

Esta investigación se realizó apoyada en el paradigma positivista, asumiendo un enfoque cuantitativo, específicamente a través de un diseño no experimental del tipo correlacional, el cual, según Hernández, Fernández y Baptista (2010) utiliza la recolección y el análisis de la información para probar hipótesis y dar respuestas a preguntas de investigación por medio del análisis numérico y estadístico. La población estuvo constituida por 90 excombatientes de las Farc-EP residentes en los municipios de Colosó y Chalán en el Departamento de Sucre, de los cuales 14 fueron pertenecientes al municipio de Chalán y 9 a Colosó. Para la medición del propósito vital se utilizó un instrumento construido por Martínez y Villaneda (2011) y validado en Colombia ese mismo año. El mismo consta de 18 afirmaciones con cuatro posibles opciones de respuesta que van desde TOTALMENTE DE ACUERDO, DE ACUERDO, EN DESACUERDO y TOTALMENTE EN DESACUERDO, valorados de $0-3$ en los ítems directos $(2,3,6,9,15)$, y de 3-0 en los inversos $(1,4,5,7,8,10,11,12,13,14,16,17,18,19,20)$. Su puntaje total ondea entre 0 y 54 , de tal manera que entre más alto sea el resultado mayor será el sentido de vida del encuestado y viceversa. Los resultados se organizan en niveles de sentido de vida de la manera siguiente: plenitud de sentido, alto sentido vital, medio sentido vital y búsqueda de sentido vital (Olivia, 2014). Cabe destacar que un puntaje en 0 no expresa ausencia de sentido de vida, ya que esta numeración no es posible conceptualmente en la realidad psicosocial de los individuos (Martínez \& Castellano, 2013).

Este instrumento tiene como base la teoría impartida por Víctor Frankl, fundamentada desde la logoterapia y su análisis existencial, y su principal objetivo es dimensionar valorativamente el sentido de vida, entendido como propósito vital y coherencia existencial, de tal forma que se identifique la vivencia y percepción del sentido de vida que tiene cada quien (Martínez \& Castellanos, 2013). Su aplicación puede ser individualizada o autoadministrable, en ámbitos de investigación o clínicos.

Cuestionario Ad Hoc. Para los aspectos social, laboral, educativo y económico se incorporó un cuestionario con preguntas cerradas diseñado por los investigadores con base en los antecedentes encontrados. Se realizaron en total 12 preguntas, las cuales fueron conformadas por 2 de nivel educativo, 5 a nivel social, 2 económico y 3 a nivel político. Asimismo, las preguntas fueron divididas entre antes de entrar el grupo armado y después de la reintegración.

Plan de análisis. Se hizo el análisis descriptivo utilizando los estadísticos descriptivos del programa SPSS; posteriormente, se realizó una comparación de medias entre las variables nominales utilizando el estadístico Chi-Cuadrado. 


\section{RESULTADOS}

Sentido de vida. Dando respuesta a uno de los objetivos de esta investigación, que se relaciona con dar a conocer en qué nivel se encuentra el sentido de vida de cada uno de los participantes, los porcentajes por niveles fueron los siguientes (Tabla 1).

Tabla 1. Sentido de Vida.

\begin{tabular}{ccccc}
\hline Plenitud Sentido & Alto Sentido Vital & Medio Sentido Vital & Búsqueda Sentido Vital & TOTAL \\
\hline 5 & 6 & 10 & 4 & 100 \\
$20 \%$ & $24 \%$ & $40 \%$ & $16 \%$ & 1005 \\
\hline
\end{tabular}

Fuente: Elaboración propia.

A partir de los resultados anteriores, se puede apreciar que el $40 \%$ de los participantes puntúan en el nivel "medio sentido vital", en el cual experimentan propósito y sentido; sin embargo, en ocasiones tienen periodos de desorientación y momentos de estancamiento (Olivia, 2014). Este nivel, tal como lo afirma la logoterapia, demuestra que sí es posible hallar un sentido al sufrimiento, puesto que, si no se puede cambiar la situación, sí es posible cambiar la actitud frente a la misma (Montoya. 2009). Seguido de un $24 \%$ de la población, el cual se encuentran en el nivel "alto sentido vital", donde la persona se siente orientada en su vida e identificada con la mayoría de los proyectos y deberes que esta representa (Olivia, 2014). El $20 \%$ de los participantes afirma estar en el máximo nivel de "plenitud de vida", llevando una vida llena de propósitos y sentido, y, por último, solo el $16 \%$ de la población se encuentra en "búsqueda de sentido vital", en este nivel las personas experimentan una vida sin sentidos ni propósito, no obstante, por momentos sí tienen una orientación.

Factores Socioeconómicos. En cuanto a los factores socioeconómicos se tomó en consideración los siguientes: haber tenido un empleo formal y acceso a la educación antes de ingresar al grupo, acceso a la educación en la actualidad, tener un empleo actualmente, sentirse acogido en la comunidad en la que residen, cómo fue su convivencia familiar antes de entrar al grupo y cómo es la misma en la actualidad. Asimismo, se tuvo en cuenta cómo califican las ayudas que les brinda el gobierno luego de haber salido del grupo y si cuentan o no con redes de apoyo en la actualidad. Los resultados están descritos en la tabla 2.

Tabla 2. Factores Socioeconómicos.

\begin{tabular}{|c|c|c|c|c|c|c|}
\hline Variable & $\mathbf{S i}$ & No & Unida & Distanciada & Buena & Mala \\
\hline Trabajo formal antes de ing. Grupo. & $48 \%$ & $52 \%$ & & & & \\
\hline Acceso a la Edu. Antes de ing. al grupo & $64 \%$ & $36 \%$ & & & & \\
\hline Acceso a la Edu. Actual & $72 \%$ & $28 \%$ & & & & \\
\hline Trabajo formal actual & $8 \%$ & $92 \%$ & & & & \\
\hline Acogido en comunidad & $72 \%$ & $28 \%$ & & & & \\
\hline Convivencia familiar antes & & & $92 \%$ & $8 \%$ & & \\
\hline Convivencia familiar actual & & & $100 \%$ & $0 \%$ & & \\
\hline Calificación ayuda gobierno & & & & & $48 \%$ & $52 \%$ \\
\hline Estigmatización & $48 \%$ & $52 \%$ & & & & \\
\hline Redes de apoyo & $72 \%$ & $28 \%$ & & & & \\
\hline
\end{tabular}

Fuente: elaboración propia

Como también se hizo necesario conocer el nivel de satisfacción de estas personas sobre el proceso de reintegración, el $60 \%$ de la población indicó sentirse satisfecho con el mismo, seguido de un $24 \%$ que manifestó un nivel regular en su satisfacción y solo el $16 \%$ indicó sentirse muy satisfecho con el proceso de reintegración. Ningún porcentaje de la población señaló un nivel insatisfecho o muy insatisfecho con el proceso de reintegración (Tabla 3). 
Tabla 3. Proceso de reintegración - Factor socioeconómico.

\begin{tabular}{cc}
\hline Muy satisfecho & $16 \%$ \\
Satisfecho & $60 \%$ \\
Regular & $24 \%$ \\
Insatisfecho & $0 \%$ \\
Muy insatisfecho & $0 \%$ \\
\hline
\end{tabular}

Fuente: Elaboración propia

Por otra parte, se cuestionó el motivo de ingreso al grupo armado y en orden jerárquico se encontraron los siguientes porcentajes: $76 \%$ de la población ingresó al grupo por motivos personales, $12 \%$ por motivos económicos, $8 \%$ por reclutamiento forzado y solo $4 \%$ por razones familiares (Tabla 4).

Tabla 4. Motivo de ingreso al grupo.

\begin{tabular}{cc}
\hline Económico & $12 \%$ \\
Reclutamiento & $8 \%$ \\
Familiar & $4 \%$ \\
Personal & $76 \%$ \\
\hline
\end{tabular}

Fuente: Elaboración propia

Y para el caso de la correlación entre el sentido de vida y los aspectos educativos, laborales, sociales y políticos de la población estudiada, se midió la misma entre los aspectos sociodemográficos como sexo, edad, estado civil, hijos, el proceso de desmovilización (reintegración), el nivel educativo y las edades de íntegro y salida de los grupos armados. Los aspectos para resaltar y que tienen mayor correlación con el propósito vital fueron: haber tenido acceso a la educación antes de ingresar al grupo armado y el tiempo que llevan los excombatientes reintegrados a la vida civil, lo cual se puede confirmar en la tabla 5.

Tabla 5. Correlación entre el sentido de vida y las características Sociodemográficas.

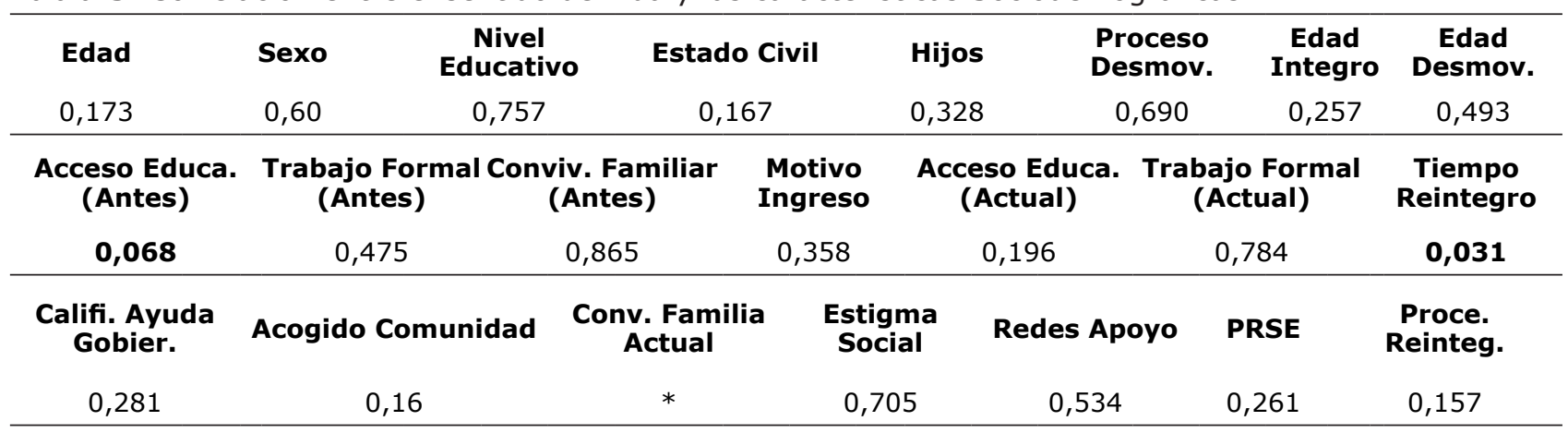

Fuente: Cálculos propios.

A partir de los resultados anteriores se puede afirmar que los participantes que sí tuvieron acceso a la educación antes de ingresar al grupo puntuaron un sentido mayor en comparación con los que no tuvieron acceso, el cual en esta investigación representa un 64\% (16 participantes) de la población estudiada, frente a un $36 \%$ el cual no tuvo acceso a la educación antes de ingresar al grupo armado. 
Asimismo, el $80 \%$ de los excombatientes afirmaron tener entre 1-3 años reintegrados a la vida civil, frente a un $20 \%$ que ha permanecido más de 3 años alejado de las armas. Este aspecto denota una característica particular, entre menos tiempo tengan de haber abandonado el grupo armado, mayores puntuaciones obtuvieron en la escala de sentido de vida.

Estos dos aspectos anteriores marcan la diferencia con el resto: haber tenido acceso a la educación antes de ingresar al grupo y tener menos tiempo reintegrados a la vida civil son los factores que más inciden directamente en el sentido de vida de la población estudiada.

Los aspectos sociales, políticos y laborales no demostraron tener incidencia en el propósito de vida de los participantes. El $72 \%$ de los participantes afirmaron sentirse acogidos en la comunidad en la que residen. Sin embargo, solo el $48 \%$ de la población sostuvieron sentirse estigmatizados por la sociedad, un aspecto que se esperaría fuera de incidencia directa en el propósito vital, pero para los participantes en esta investigación resultó no serlo de manera efectiva. De manera similar, un $52 \%$ de la población negó sentirse apoyado por la Política Nacional de Reintegración Social y Económica para Personas y Grupos Armados Ilegales (PRSE) en la construcción de su proyecto de vida, es igual al porcentaje de los participantes que sostuvieron estar insatisfechos con la ayuda que el gobierno les ha brindado para la obtención de un empleo formal. Sin embargo, el 16\% de la población afirmó sentirse muy satisfecho con su proceso de reintegro a la vida civil, seguido de un $60 \%$ que manifestó sentirse satisfecho con esto y un $24 \%$ que puntuó una calificación neutra en este ítem. Esto nos demuestra que ninguna persona encuestada afirma no estar satisfecho con su proceso de reintegración a la vida civil.

Siguiendo con los aspectos sociales (en el cual se incluyó su núcleo familiar), el $72 \%$ sostuvo contar con redes de apoyo significativas en el reintegro a su vida civil, aspecto que se podría relacionar con la convivencia familiar actual, en el cual el $100 \%$ de la población afirmó contar con una familia unida actualmente, a diferencia del $8 \%$ que manifestaron tener una relación distante con sus familiares antes de ingresar al grupo, aspecto que notablemente mejoró con el proceso de reintegración.

Por último, frente al trabajo formal antes de entrar al grupo y actualmente, se demostró que el $52 \%$ de la población no contaba con un trabajo formal antes del ingreso al grupo, y el 92\% de los excombatientes actualmente no poseen un medio de obtención de dinero formal. Sin embargo, no es coherente con los porcentajes obtenidos en el ítem de calificación al gobierno para la ayuda de un empleo formal, puesto que el $48 \%$ respondieron sentirse satisfechos, frente a un $52 \%$ que afirma no estarlo.

\section{DISCUSIÓN Y CONCLUSIONES}

Este estudio permitió identificar las diferentes perspectivas que tienen los excombatientes sobre su reintegro a la vida civil, sobre cómo ven y sienten cada uno al haber pertenecido a la guerra, y determinar sus disímiles actitudes frente a la vida luego de haber pasado por una etapa determinante. Como también, conocer los aspectos que posiblemente se asocian al propósito de vida nos proporciona una correlación para poder definir este mismo.

Estos datos indican que el propósito de vida es similar a los resultados hallados en la investigación de Matallana y Villaneda en 2013, en la cual se encontró que los participantes de la investigación tenían un proyecto de vida concreto, ya que manifiestan de una forma acorde y organizada sus recursos y posibilidades para alcanzar las metas que quieren en su vida. En la presente investigación, la mayoría de los participantes se encuentran en niveles altos de sentido de vida, en donde expresan claramente lo que quieren, conociendo y admitiendo sus posibilidades, recursos y medios para la obtención de este.

Sin embargo, y a pesar de todo, a los resultados se suman los sentimientos de estigmatización que suelen tener los excombatientes por parte de la sociedad en la cual se reintegraron, muchos de los participantes afirmaron sentirse estigmatizados en la comunidad donde viven, aspecto que resulta preocupante para la reintegración integral de estas personas a la sociedad, puesto 
que, tal como lo afirma Cabrera (2016), la estigmatización puede determinar los planes de las personas en proceso de reintegración. A pesar de esto, resulta paradójico las posiciones de los participantes al manifestar que se sienten acogidos en la comunidad donde residen, ya que, al hablar de estigma, se está tratando la mirada social con la cual son percibidos por la comunidad que los rodea, razón por la cual resulta incongruente que se sientan acogidos en una comunidad que los estigmatiza.

Tal como lo expresan Rodríguez, Yunis \& Girón (2016) "las redes de apoyo cumplen un papel de acompañamiento y son facilitadoras de ese proceso de transición hacia la vida civil" ( $\mathrm{p}$. 121). En esta investigación se estudió la presencia de las redes de apoyo en los excombatientes participantes, el $72 \%$ afirmaron contar con dichas personas significativas, el cual, aunque no fue una incidencia directa en la variable de propósito de vida, la gran mayoría afirmo contar con algún familiar, amigo, o profesional que contribuyan al bienestar de estos mismos en el proceso de reintegración.

Siguiendo la posición planteada por Matallana y Villaneda (2013), la sociedad debe aportar para que el tejido social se reconstruya, partiendo de la premisa que las personas excombatientes abandonan las armas para vivir en una sociedad bajo el marco de la legalidad, todas las personas de la sociedad deben dejar a un lado los estereotipos que representa ser un excombatiente, y acoger a estos de manera satisfactoria para que los propósitos de vida de las personas excombatientes pueda cumplirse de manera más completa y lograr una posible reconciliación de partes.

Siendo congruentes con los resultados obtenidos en la investigación, se hace necesario destacar la importancia de un proceso educativo eficaz en esta población vulnerable a ingresar a cualquier grupo armado, la relación entre haber podido acceder a un grupo armado y su sentido de vida actual, luego de haberse reintegrado, es muy directa. Sumado a esto, los comentarios y percepciones que tienen algunas personas en la investigación de Rueda (2008) comprueban que el propósito de vida si está ligado a la educación que pueden acceder o que han tenido, manifestando que, gracias a la educación, se desarrollan capacidades y habilidades para adentrarse y fortalecerse autónomamente en la vida civil a la cual se enfrentan.

Similar a esto, se resalta la relevancia de un proceso de desarme colectivo y con garantías para estas personas permite que estas mismas eleven sus esperanzas en un futuro, tengas proyecciones, propósitos y sentido en la vida. Siendo esta la variable con mayor correlación, seguida del tiempo que llevan los participantes estando reinsertados, sería interesante realizar una investigación de manera más minuciosa sobre estos aspectos. Conocer cuáles son los motivos por los cuales se guían en tener esta relación, abriría un camino más para la inclusión de estas personas a la educación desde la temprana edad.

Visto que solo el $8 \%$ de los participantes de la investigación cuentan con un empleo formal, parece incongruente afirmar que solo el $52 \%$ de la población investigada no está conforme con las ayudas brindadas por el Gobierno para la obtención de un empleo formal. Esta afirmación se comprueba con los resultados obtenidos en este aspecto; sin embargo, los resultados son similares a lo afirmado por Rendón (2017) al sostener que sólo el $20 \%$ de los desmovilizados obtienen un empleo formal. Por esto, se hace necesario invitar a las empresas de la región a incluir a esta población dentro de esta para contribuir con la responsabilidad social de las mismas.

Por otra parte, conocer las motivaciones que llevaron a los participantes a ingresar al grupo, se evidenció de manera análoga con lo manifestado por Rodríguez, Romero, Acuña y Díaz (2009) puesto que el $76 \%$ de los participantes afirmaron haber ingresado al grupo por motivos personales, que podrían relacionarse con la frustración de no hacer posible el cambio de las injusticias sociales, algún sentimiento de venganza o la remuneración económica que implica pertenecer al grupo.

Las diferencias entre los excombatientes residentes en Colosó y Chalán son significativas, más que todo en la percepción que tienen cada uno hacia el acuerdo firmado por ellos en 2016, con respecto a sus proyectos productivos, los participantes en Chalán afirmaron estar más proactivos en la obtención de lo prometido en el Acuerdo Final. Los participantes residentes en Chalán 
se mostraron más activos y motivados por realizar proyectos productivos que los impulsen a alcanzar sus metas y propósitos. Lo cual sería similar a lo expresado por un participante de la investigación llevada a cabo por la ACR (2008) donde este expresa "De pronto yo opino que necesitamos trabajar, no plata regalada, sino queremos es trabajar y ganárnosla con el sudor de la frente" (p. 5).

Siguiendo lo anterior, se propone la necesidad de investigar el porqué de estas diferencias pronunciadas por los participantes de los distintos municipios, siendo vecinos en la misma región y con similares condiciones de vida.

Además, los aspectos sociales, políticos y laborales no demostraron tener incidencia en el propósito de vida de los participantes. El $72 \%$ de los participantes afirmaron sentirse acogidos en la comunidad en la que residen. Sin embargo, solo el $48 \%$ de la población sostuvieron sentirse estigmatizados por la sociedad, un aspecto que se esperaría fuera de incidencia directa en el propósito vital, pero para los participantes en esta investigación resultó no serlo de manera efectiva.

A manera de conclusión, haber sido parte de un grupo armado al margen de ley representa para la persona muchos cambios a nivel emocional y mental. Conocer el propósito de vida que estos tienen luego de haber hecho el sacrificio psicológico de cambiar de contextos en los cuales se desenvuelven, es de vital importancia para la reconstrucción de la paz en el país, la sociedad y los excombatientes tiene la responsabilidad de llevar esto a cabo, así como el Gobierno de brindar las herramientas y estrategias para que esto se cumpla. Como también se cuestiona la percepción que tienen estos participantes hacia el Acuerdo firmado; conocer cómo ven su futuro luego de haber accedido a terminar la guerra, abre una línea de investigación para evitar o disminuir la reincidencia de estos a cualquier grupo armado al margen de la ley. Las acciones que se realizan en la sociedad día a día también influyen en la decisión de las personas que comúnmente son marginadas al querer pertenecer a un grupo armado, que bien sea por violencia o por fines de bien común, son una medida de seguridad que toman ciertas personas al enfrentarse a los miedos e incertidumbres que repercuten por vivir en una sociedad de estigmas y desigualdades.

\section{REFERENCIAS}

Agencia Colombiana para la Reintegración (ACR) (2008). Los excombatientes proponen: aportes y percepciones sobre la reintegración en Colombia. Recuperado de http://www.urosario. edu.co/Mesas-de-liberacion/Documentos/7-Los-excombatientes-proponen-aportes-ypercepcion/

Agencia Colombiana para la Reintegración. (2016a). Evolución del Proceso de Reintegración, Fortaleza Institucional basada en la experiencia y lecciones aprendidas. Recuperado de http:// www.reintegracion.gov.co/es/la-reintegracion/centro-de-documentacion/Documentos/ Evoluci\%C3\%B3n\%20del\%20Proceso\%20de\%20Reintegraci\%C3\%B3n\%20Fortaleza\%20 Institucional\%20basada\%20en $\% 201 a \% 20$ experiencia $\% 20 \mathrm{y} \% 20$ lecciones $\% 20$ aprendidas.pdf

Agencia colombiana para la Reintegración. (2016b). Caracterización de usuarios, personas en proceso de reintegración. Recuperado de https://www.reintegracion.gov.co/es/atencion/ SiteAssets/Caracterizacion\%20usuarios.PDF

Bravo, P. (2016). Así se protege a un desmovilizado en Colombia. CNN. Recuperado de https:// cnnespanol.cnn.com/2016/05/04/asi-se-protege-a-un-desmovilizado-en-colombia/

Cabrera, D. (2016). Las redes sociales como entornos protectores frente a la estigmatización de los jóvenes en proceso de reintegración en la ciudad de Bogotá durante el año 2016. Tesis de Magister. Pontificia Universidad Javeriana, Bogotá. 
De la Espriella, R., \& Falla, J. (2009). Reflexiones sobre la atención en salud mental de desmovilizados de grupos armados en Colombia. Revista Colombiana Psiquiatría. 38 (2). 230-247.

Frankl, V. E. (1988) EI hombre en busca de sentido. Barcelona: Herder (original de S Y 1946).

Hernández, R., Fernández, C., \& Baptista, P. (2010). Metodología de la Investigación. México: McGraw Hill.

Instituto de Estudios Geoestratégicos y Asuntos Políticos. (2013). Desarme, desmovilización y reintegración, DDR: una introducción para Colombia. Recuperado de https://iugm.es/wpcontent/uploads/2017/02/Cuaderno-An\%C3\%A1lisis-DDR.pdf

Lara, L. (2016). ¿Y después de la guerra qué? Avatares en el tránsito a la vida civil de jóvenes desmovilizados de las Farc. Universitas Humanísticas, (82), 49.73.

Lara, L., \& Delgado, R. (2010). Trasegar de las subjetividades y las memorias de las y los jóvenes desmovilizados en el tránsito a la vida civil. Una mirada a los programas educativos y de apoyo psicosocial. Universitas Humanística, (70), 29-56.

Martínez, E., Castellanos, C. (2013). Percepción de Sentido de Vida de Universitarios Colombianos. Pensamiento Psicológico, 11(1), 71-82.

Martínez, E., Trujillo, A., Día, J. \& Osma, J. (2011). Desarrollo y estructura de la escala dimensional del sentido de vida. Acta Colombiana de Psicología. 14, (2): 113-119.

Matallana, J., Villaneda, F. (2013). La comprensión del sentido de vida de personas que hicieron parte de la insurgencia y participaron en procesos de negociación política con el Estado Colombiano.

Mejía, L. (2014). La Reintegración social y Económica de los Grupos Armados ilegales en Colombia: Reflexiones a partir de la Trayectoria de Nueve Excombatientes. Tesis de Maestría, Universidad Colegio Mayor de Nuestra Señora del Rosario, Bogotá. Recuperado de http:// www.reintegracion.gov.co/es/la-reintegracion/centro-de-documentacion/Documentos/ La\%20Reintegraci\%C3\%B3n\%20social\%20y\%20econ\%C3\%B3mica\%20de\%20los\%20 grupos\%20armados\%20ilegales\%20en\%20Colombia.pdf

Mejía, L. (2014). La Reintegración social y Económica de los Grupos Armados ilegales en Colombia: Reflexiones a partir de la Trayectoria de Nueve Excombatientes. Tesis de Maestría, Universidad Colegio Mayor de Nuestra Señora del Rosario, Bogotá. Recuperado de http:// www.reintegracion.gov.co/es/la-reintegracion/centro-de-documentacion/Documentos/ La\%20Reintegraci\%C3\%B3n\%20social\%20y\%20econ\%C3\%B3mica\%20de \%20los\%20 grupos\%20armados\%20ilegales\%20en\%20Colombia.pdf

Montoya, J. (2009). Sentido de vida en adolescentes desplazados por la violencia en Colombia: avances tesis doctoral [en línea]. Presentado en $3^{\circ}$ Congreso Latinoamericano de Logoterapia y Análisis Existencial: Educación, Familia y Salud. Universidad Católica Argentina, Centro de Logoterapia y Análisis Existencial, Buenos Aires, Argentina.

Noblejas de la Flor, M., \& Rodríguez, M. (2005). Logoterapia, una ayuda para afrontar la adversidad. Revista de Psicología, Ciències de l'Educació i de l'Esport, 6, 105-112.

Olivia, S. (2014). Dimensiones de sentido de vida en estudiantes de la carrera de licenciatura en psicología clínica y licenciatura en psicología industrial/organizacional de 5to. Año de la universidad Rafael Landívar. Tesis de grado. Guatemala de la asunción. Recuperado de http://recursosbiblio.url.edu.gt/tesisjcem/2014/05/22/Oliva-Silvia.pdf 
Martinez-Ballutt et al - Asociación entre el Propósito de Vida y los Factores Socioeconómicos de Excombatientes

Radiónica (2017). ¿Cuál es la diferencia entre los tres términos?, ¿cuál de estos aplican en el proceso de paz actual entre el Gobierno y las Farc-EP? Recuperado de https://www.radionica. rocks/noticias/reinsercion-reincorporacion-y-reintegracion

Redón, O. (2017). Reintegración en Colombia ha sido efectiva en 76\%. El Colombiano. Recuperado de http://www.elcolombiano.com/colombia/reintegracion-en-colombia-ha-sido-efectiva-en76-IE6767921

Rendón, O. (2018). De los desmovilizados, sólo el $20 \%$ encuentra empleo formal. El Colombiano. Recuperado de https://www.elcolombiano.com/colombia/paz-y-derechos-humanos/empleoformal-para-desmovilizados-en-colombia-IE8556291

Rodríguez, L., Yunis, K., \& Girón, C. (2015). Resignificación del sentido de la vida de personas desvinculadas y desmovilizadas del conflicto y contribución de las redes de apoyo en su transición hacia la vida civil. Informes Psicológicos, 15(1), pp. 105-126. http://dx.doi. org/10.18566/infpsicv15n1a06

Rodríguez, T., A. Romero, Acuña, N. R., \& Díaz, I.,L. (2009). Factores psicosociales que inciden en la reintegración social de tres reclusos con vínculos a los grupos armados ilegales (FarcEP, UC-ELN y AUC) del centro penitenciario y carcelario de villahermosa. Pensamiento Psicológico, 6(13), 219-238.

Rueda, M. (2008). Reinserción y postconflicto: el "volver" de jóvenes excombatientes a la vida civil. (Tesis de pregrado). Universidad del Valle. Cali.

Sennett, R. (2018). El respeto: (Sobre la dignidad del hombre en un mundo de desigualdades). España: Editorial Anagrama. 304 p

Sennett, R. (2018). El respeto: (Sobre la dignidad del hombre en un mundo de desigualdades). España: Editorial Anagrama.

Somaye, R., Sohrabi, F., Shahrak, M., Talepasand, S., Nokani, M., \& Hasani, M. (2015). The Effect of Group Logotherapy on Meaning in Life and Depression Levels of Iranian Students. International Journal for the Advancement of Counselling. 37 (1), 54-62. DOI: https://doi. org/10.1007/s10447-014-9225-0 